Full length article

\title{
Deformation behavior of nano-porous polycrystalline silver. Part II: Simulations
}

\author{
S. Zabihzadeh a, b, J. Cugnoni ${ }^{\text {c }}$, L.I. Duarte ${ }^{\text {d }}$, S. Van Petegem a, H. Van Swygenhoven a, b, * \\ a Photons for Engineering and Manufacturing, SYN, Paul Scherrer Institut (PSI), CH-5232 Villigen, Switzerland \\ ${ }^{\mathrm{b}}$ Neutrons and Xrays for Mechanics of Materials, Institute of Materials, École Polytechnique Fédérale de Lausanne, CH-1015 Lausanne, Switzerland \\ ${ }^{\mathrm{c}}$ Ecole Polytechnique Fédérale de Lausanne (EPFL), LMAF-STI, Lausanne, CH-1015, Switzerland \\ ${ }^{\mathrm{d}}$ ABB Switzerland Ltd. Corporate Research, $\mathrm{CH}-5405$, Baden-Daettwil, Switzerland
}

\section{A R T I C L E I N F O}

\section{Article history:}

Received 1 September 2016

Received in revised form

15 February 2017

Accepted 8 April 2017

Available online 20 April 2017

\section{Keywords:}

Finite element simulations

Porous structure

Size effects

Deformation behavior

\begin{abstract}
A B S T R A C T
Three-dimensional finite element simulations of nano-porous silver structures are performed to understand the correlation between the porous morphology and the mechanical behavior. The nanostructures have been obtained from ptychographic X-ray computed tomography. The simulations allow distinguishing between the interplay and role of the ligament size, the pore morphology and the porosity, and therefore provide a better comprehension of the experimental observations. We show that the proposed model has a predictive character for mechanical behavior of nano-porous silver.

@ 2017 Published by Elsevier Ltd on behalf of Acta Materialia Inc. This is an open access article under the
\end{abstract} CC BY-NC-ND license (http://creativecommons.org/licenses/by-nc-nd/4.0/).

\section{Introduction}

The mechanical response of porous materials can be modeled with finite element (FE) simulations [1]. Several models exist to generate these structures such as the periodic Kelvin cells [2] and the Weaire-Phelan cells [3] generated with the surface Evolver program developed by Brakke [4] or the Voronoi Diagram [5]. It is however also possible to generate a micromechanical model directly from the microstructure obtained by tomography techniques [1]. To capture the effects of heterogeneity and structure of the porous network three-dimensional (3D) simulations are required. Recent developments in high-resolution 3D imaging techniques, such as focus ion beam (FIB)/scanning electron microscopy (SEM) or X-ray tomography launched a larger interest in performing $3 \mathrm{D}$ simulations on the actual microstructure of the porous materials [6-12]. Using high-resolution 3D images obtained from serial-block face scanning electron microscopy [13], Carr et al. [9] studied the influence of aging on the porosity, the pore distribution and the elastic modulus of sintered micro silver paste. It was reported that aging does not influence the global density and the

\footnotetext{
* Corresponding author. Photons for Engineering and Manufacturing, SYN, Paul Scherrer Institut (PSI), CH-5232 Villigen, Switzerland.

E-mail address: helena.vs@psi.ch (H. Van Swygenhoven).
}

elastic modulus of the material. However, aging increases the heterogeneity in the pore distributions and results in a clustering of pores leading to a local decrease of the elasticity next to the high porous regions. Our previous [14] experimental work on nanoporous sintered silver layers showed a strong dependency of the mechanical behavior on the microstructure. To allow a better understanding, 3D (FE) microstructure-based simulations are performed. The statistically representative volume elements or RVE were obtained by ptychographic tomography, a technique providing a higher resolution than X-ray nano-tomography [15]. By comparing the simulation results with in-situ and ex-situ tensile tests the deformation mechanisms are discussed.

\section{Experimental setup and simulation methodology}

\subsection{Sample fabrication}

Eight thin layers $(25 \pm 5 \mu \mathrm{m})$ of porous polycrystalline silver layers are produced during pressure assisted sintering. The samples are called as S1-S8 and are sintered in the temperature range of 210 $-300{ }^{\circ} \mathrm{C}$, pressure range of 4-12 MPa during 3-10 min. More details about sample fabrications are found in first part of the paper [15]. S1 and S8 are samples sintered respectively at lowest and highest extremes of sintering conditions. 


\subsection{In-situ tensile testing}

The in-situ mechanical tests are carried out at the powder diffraction station of MS beam-line of the SLS [16]. For the in-situ experiments, an X-ray beam with energy of $20 \mathrm{KeV}$ and a size of $400 \times 200 \mu \mathrm{m}^{2}$ is selected and the diffraction patterns are recorded with the MYTHEN II microstrip detector [17]. The diffraction peaks are fitted with a split Pearson-VII function plus a linear function (for background noise) and information about full-width-at halfmaximum (FWHM), integral width (iw) and peak position $(\theta)$ are extracted. Finally, Williamson-Hall $(\mathrm{W}-\mathrm{H})$ method based on Gaussian-Cauchy distribution [18] is applied for deconvolution of the peak broadening caused by the grain size and root-meansquare (RMS) strain $[14,19,20]$. RMS strain is defined as the spread of the lattice strain from the averaged value inside a grain, calculated from FWHM, and is an estimation of the internal strain inside the material. A dog-bone shaped geometry with width of $2000 \pm 5 \mu \mathrm{m}$, thickness of $25 \pm 5 \mu \mathrm{m}$ and gauge length of $1500 \mu \mathrm{m}$ is selected for tensile experiments and strain is measured using digital image correlation (DIC) method. The specimens are deformed during continuous tensile test at room temperature and with a strain rate for $0.015 \% . \mathrm{s}^{-1}$ for ex-situ and $0.01 \% . \mathrm{s}^{-1}$ for in-situ experiments.

\subsection{Simulation methodology}

The general framework of the microstructure-based simulation is similar to the work done by Maleki et al., 2016 [23]. First, the high-resolution 3D reconstructed images obtained from the ptychography measurements [15] are segmented for the silver phase using the ITK-SNAP software, then they are meshed using the software VoxelMesher developed at the Laboratory of Applied Mechanics and Reliability Analysis (LMAF) group of École polytechnique fédérale de Lausanne based on the algorithm presented in Ref. [21]. A quadratic ten-node tetrahedron is selected as the meshing element. Details about the X-ray ptychography measurement and analyzed porous structure of the samples are found in Ref. [15]. Fig. 1 displays a representative 3D image of the segmented silver phase for a pillar with diameter of $4 \mu \mathrm{m}$. The FE package used in this work is Dassault Simulia Abaqus Standard using an implicit integration scheme (using the standard direct Newton-Raphson nonlinear solver). A mesh convergence analysis was performed to verify the stagnation of the solution with mesh refinement levels (convergence within $0.5 \%$ ).
To model the loading, a displacement controlled mixed statickinematic boundary condition is selected. This boundary condition is chosen based on the work of Cugnoni and Galli (2010) [22] on particle-reinforced composite materials; one end of the object is kept fixed in the loading direction while a displacement ramp up to $1 \%$ effective strain is imposed on the other end parallel to the tensile direction, the perpendicular lateral faces are left stress-free. More details about these boundary conditions are explained in Ref. [23]. A RVE approach [22,23], is applied for the homogenization to determine the smallest volume representative for the continuum behavior. The bulk material properties of the silver skeleton, including its elastoplastic behavior, are identified using an inverse homogenization approach on a selected sample; S5. The accuracy of the model is further verified by comparing the simulation with experimental results obtained from ex-situ tensile deformation with a strain rate of $0.015 \% . \mathrm{s}^{-1}$ performed at room temperature on a wide set of sintered silver layers. In what follows all simulations results are compared with experiments performed at a strain rate of $0.015 \% . \mathrm{s}^{-1}$ except stated otherwise.

\subsection{Representative volume element}

Due to heterogeneity of the porous structure, the FE simulation of the porous materials should be performed in three dimensions [24]. 3D simulations are however expensive in terms of computational time. To overcome this issue a homogenization approach is applied to minimize the required volume for the simulations. In this work the RVE approach proposed by Cugnoni and Galli [22] is employed. RVE refers to the smallest volume extracted from the bulk of a heterogeneous material which is large enough to be representative of the behavior of the whole sample while it is small enough to be simulated or measured in a reasonable time and cost [25]. According to De Gol et al. [26] an appropriate size of the RVE for an object with porosity in the range of $25-30 \%$ is estimated to be around of 8-9 times the averaged pore size. For this work, sample S5, which has a porosity of $29 \%$ and pore size in the 200-400 nm range, is selected as the reference sample for RVE identification and a cube is considered as the proper shape due to the overall isotropy of the microstructure. Details on the porous structure of sample S5 are found in Ref. [15]. The RVE size is verified by studying the convergence of the results when changing the size of the cube, by verifying the position invariance of the solution and by comparing the scatter within multiple realizations. Therefore, three different cubes with side length of $2.5 \mu \mathrm{m}, 3 \mu \mathrm{m}$ and $4 \mu \mathrm{m}$ are
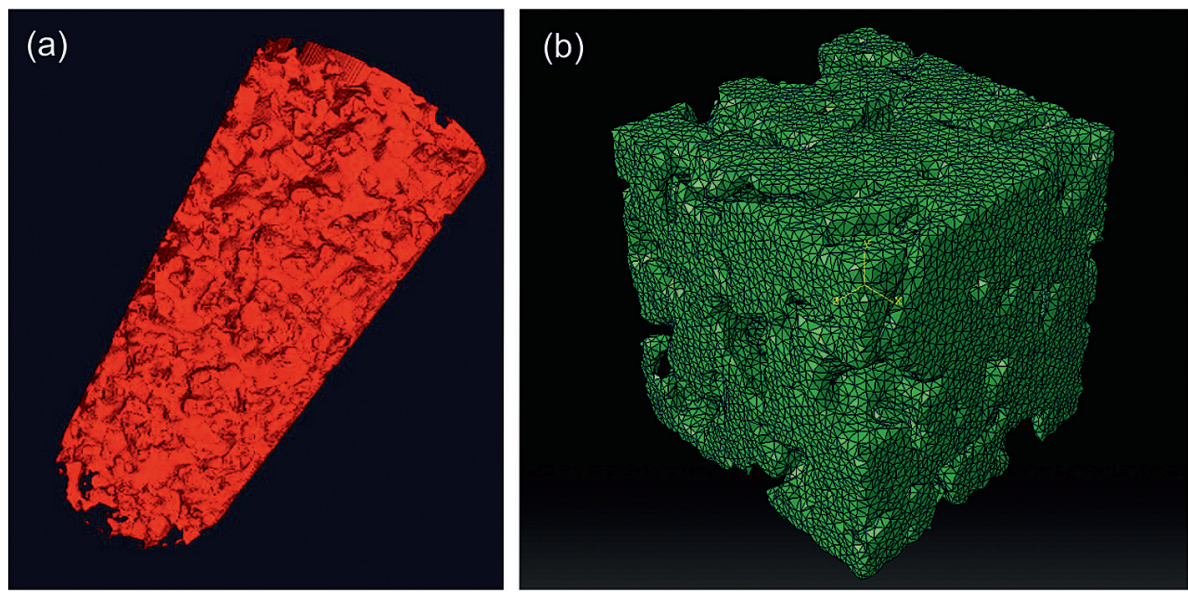

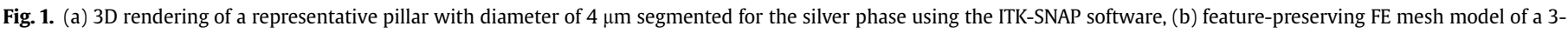
$\mu \mathrm{m}$-length cube extracted from the pillar presented in (a). 
extracted from sample S5 for the first series of the simulations. All selected cubes contain $29 \%$ porosity. Fig. 2a displays the simulated effective stress-strain curves of these RVEs. A scattering around 11\% is observed between the results of $2.5 \mu \mathrm{m}$ and $3 \mu \mathrm{m}$-length cubes, while the curves of $3 \mu \mathrm{m}$ and $4 \mu \mathrm{m}$-length cubes converge with a scattering less than $3 \%$. The experimental stress-strain data is also presented as scatter curve (purple sphere) in the graph. Fig. 2b compares the simulated stress-strain values for three different $3 \mu \mathrm{m}$-length cubes chosen within a pillar. The data converges with a scattering less than $3 \%$ suggesting a $3-\mu \mathrm{m}$-length cube as an optimum size for RVE. Fig. $1 \mathrm{~b}$ presents a representative featurepreserving FE-mesh model of a $3-\mu$ m-length cube.

\subsection{Material properties: inverse homogenization method}

The mechanical behavior of bulk silver (with a microstructure similar to the porous materials in terms of the grain size and defect structure) serves as input for the simulations. In this work the apparent elastoplastic response of bulk polycrystalline silver is identified using an inverse homogenization method [27]. In this approach, the properties of the porous material are known at the macro scale, and the mechanical behaviors of the bulk materials in the porous skeleton is to be determined [27]. Note that this approach is mainly used to obtain the apparent elastoplastic properties of the polycrystalline silver, while the elastic parameters such as Poisson's ratio and Young's modulus are selected from reported values in the literature [28]. For this simulation model, an isotropic elastoplastic response with von-Mises plasticity without creep (rate independent) is considered for the silver material. To simplify the model the influence of the grain structure, grain boundary and defects are excluded. The main objective of the proposed model is to determine the influence of the porous morphology in the deformation behavior of the silver layers. The elastoplastic response of bulk silver $\left(\sigma_{\mathrm{y}}\right)$ is described with a power law hardening relation presented in equation (1) [29]. Here, $\sigma_{0}$ is the yield stress, $\mathrm{c}$ and $\mathrm{n}$ are respectively the strength index and the strain hardening coefficient. A plastic strain $\left(\varepsilon_{\mathrm{p}}\right)$ in the range of $0-0.1$ is selected.

$\sigma_{y}=\sigma_{o}+C \varepsilon_{p}^{n}$

The simulations for parameter identification are performed on sample S5, with a porosity of $29 \%$ and an RVE equal to $3-\mu$ m-length cubes. The procedure starts by estimating the fitting parameters $\left(\sigma_{0}, c\right.$ and $\left.\mathrm{n}\right)$ and calculating $\sigma_{\mathrm{y}}$ from the estimated values using equation (1). Then, the simulation is run using calculated $\sigma_{\mathrm{y}}$ (as input for the material properties) and the simulated effective stress-strain output is compared with the experimental data. The identification loop continues until a good agreement between the simulation and experiment is achieved. Fig. 3 displays the frame work of the inverse-homogenization method. Table 1 lists the optimum parameters for the apparent elastoplastic response of the bulk silver in the current model. Here "E" and " $v$ " are respectively the identified elastic modulus and Poisson's ratio of the porous skeleton.

Fig. 4 compares the experimental and simulated apparent stress and strain curves using the parameters of Table 1 . A good agreement is observed between the experiment and simulation in the elastic and the plastic regimes.

\section{Results}

The experimental stress-strain curves of samples S1, S8 (the two extremes in terms of sintering parameters) and S5 (sintered with intermediate conditions) are presented in Fig. 5. The samples are deformed at room temperature during continuous tensile deformation with a strain rate of $0.015 \% . \mathrm{s}^{-1}$. A similar ultimate strain (around 1\%) is measured for all samples, the flow stress is however different. S1, with a porosity of 47\% [15] breaks at a stress value of $30 \mathrm{MPa}$, while S5 and S8 with resp. porosity of $29 \%$ and $22 \%$ [15] reach $85 \mathrm{MPa}$ and $120 \mathrm{MPa}$.

A series of simulations are performed on $3 \mu \mathrm{m}$-length cubes

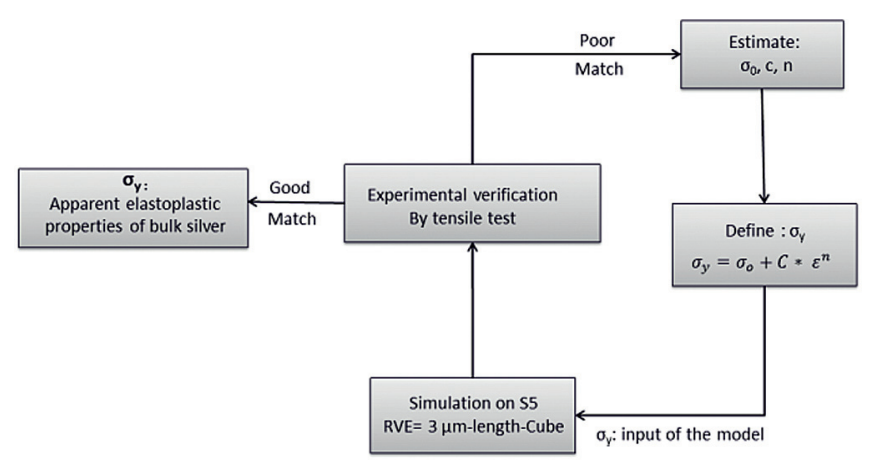

Fig. 3. Framework of the inverse homogenization approach applied for FE simulation model in order to identify the apparent elastoplastic properties of the bulk silver.
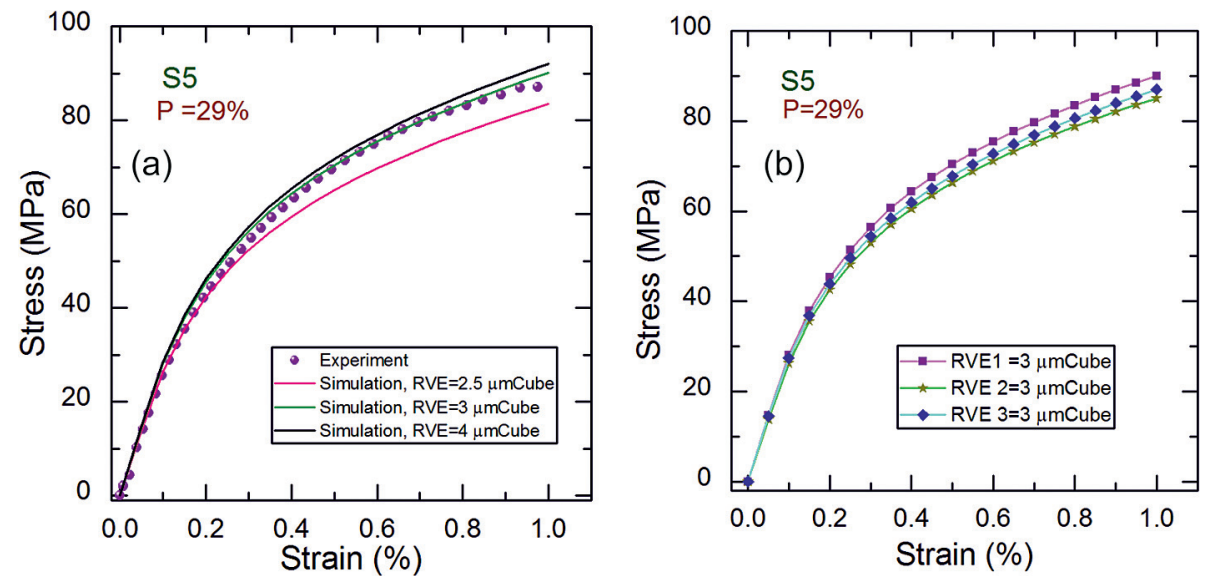

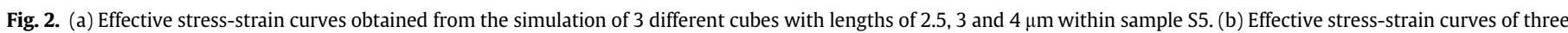
$3-\mu \mathrm{m}$-length cubes extracted from different parts of pillar S5. All RVEs are composed of $29 \%$ porosity. 
Table 1

Identified elastoplastic response of polycrystalline bulk silver used as input for the simulation.

\begin{tabular}{llllll}
\hline Fitting parameters & $\sigma_{0}(\mathrm{MPa})$ & $\mathrm{C}(\mathrm{MPa})$ & $\mathrm{n}$ & $\mathrm{E}(\mathrm{GPa})$ & $v$ \\
\hline$\sigma_{\mathrm{y}}=\sigma_{0}+\mathrm{C}^{*} \varepsilon^{\mathrm{n}}$ & 80 & 900 & 0.39 & 70 & 0.37 \\
\hline
\end{tabular}

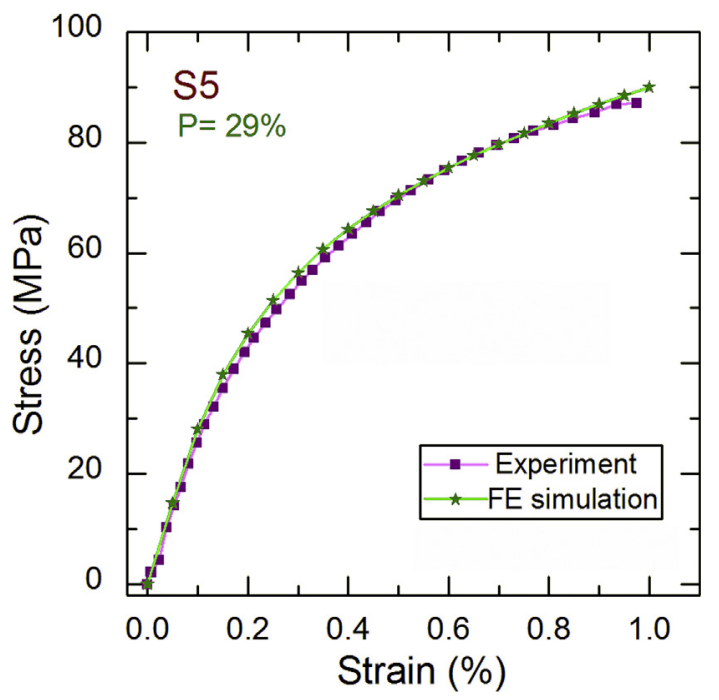

Fig. 4. Comparison between the experimental and simulated stress-strain curves for sample S5 deformed during tensile testing. The values of identified elastoplastic response in Table 1 are used as the material properties for the simulation.

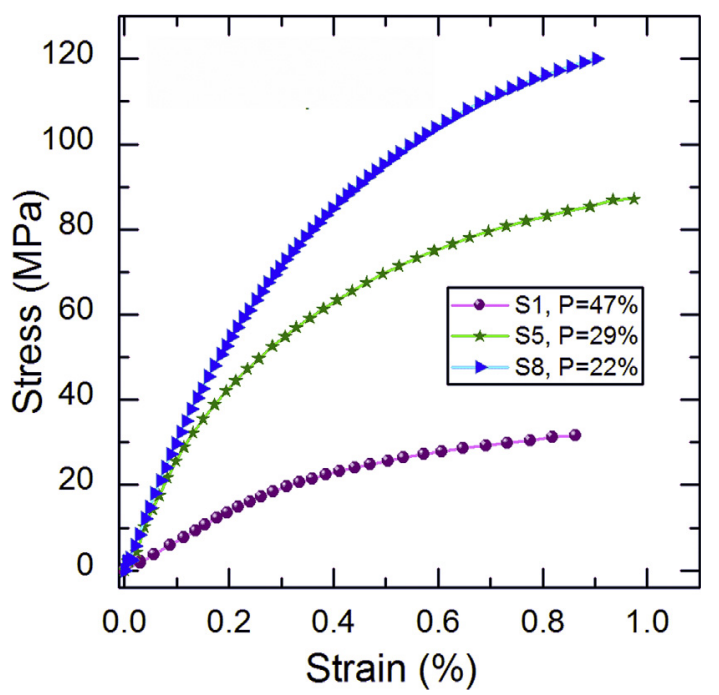

Fig. 5. Experimental stress-strain curves of samples S1, S5 an S8 deformed during continuous tensile testing.

extracted from different regions of samples S1, S5 and S8. Fig. 6a displays the effective stress-strain curves of the samples obtained from the experiment (scatter curves) and simulations (full line curves). The simulation data of sample S1 have a standard deviation around $4 \mathrm{MPa}$ which is $11 \%$ of the averaged values. Maximum deviation from the experimental curve amounts $36 \%$. This scatter is mainly due to the heterogeneity of the porous structures. Differences in the elastic regime are observed. The model is found systematically stiffer than the experimental material. This could be related to the influence of the creep which is not considered in the model and can have an influence at the rather slow experimental loading rates. A smaller standard deviation (around $2 \mathrm{MPa}$ or $1 \%$ of the averaged value) is observed between the simulated curves of sample S8, which denotes a better homogeneity. On the other hand, the calculated ultimate stress from simulations are $(\approx 10 \%)$ smaller than the experimental data and the proposed model underestimates the flow stress of this sample. Since the RVE and material properties are identified by performing series of simulations on sample S5, the scattering between the simulated curves and the maximum deviation from the experimental stress-strain data is less than $3 \%$ for this specimen. To estimate the accuracy of the proposed model, the values for the simulation scattering should be compared with the experimental dispersion. For this purpose, several tensile tests are performed for each sample and the experimental scatter is determined from the standard deviation of the measured ultimate stresses. A scatter of $35.4 \%, 11.4 \%$ and $11 \%$ is observed respectively for the experimental data of samples S1, S5 and S8. The experimental scatter is mainly attributed to heterogeneous structure of the silver layers. The results are shown as error bars in Fig. 6. A smaller scatter is observed for the simulations compared to the experiments. In Fig. $6 \mathrm{~b}$ the simulated and experimental stress values at a strain of $0.9 \%$, close to failure, are presented for each sample as a function of its porosity. The corresponding stress values obtained from the simulations range again well within the experimental scatter presented as error bar.

Simulations are further performed on the deformation behavior of five other samples, namely S2, S3, S4, S6 and S7, with porosity of $45 \%, 41 \%, 35 \%, 33 \%$ and 32\%, respectively. In Fig. 7 the experimental (black cross) and simulated (red circle) values of the stress at a strain of $0.8 \%$ are plotted as a function of the pore volume fraction of the samples. For samples S1, S5 and S8 the average stress obtained for several simulations using different RVEs are shown. A good agreement between the simulation and experiment is observed. As expected, the stress values for both experiment and simulation decrease with increasing porosity. Fig. $7 \mathrm{~b}$ displays the simulated and experimental values of the apparent yield stress ( $0.2 \%$ plastic strain) of the specimens as function of their relative density; $\rho^{*} / \rho=1-\mathrm{P}$, in which $\rho^{*}$ denotes the density of the porous material, $\rho$ the density of the bulk silver layer and $P$ the porosity obtained from ptychographic-tomography measurements [15]. The averaged value of stress obtained from at least two valid tensile tests has been plotted for all samples.

An increase in the yield stress is observed by increasing the relative density $\left(\rho^{*} / \rho\right)$. The tensile stress at a strain of $0.8 \%$ and yield stress of the samples obtained from simulations deviates from the corresponding experimental values by respectively $17 \%$ and $3 \%$ for $\mathrm{S} 1,5 \%$ and $5 \%$ for S2, $15 \%$ and $2 \%$ for S3, $14 \%$ and $5 \%$ for S4, less than $1 \%$ for S5, $2 \%$ and $15 \%$ for S6, $8 \%$ and $3 \%$ for S7, and $8 \%$ and $12 \%$ for S8. The good correlation between the simulation and the experiment suggests that, despite its simplifications (isotropy, no grain boundaries, no creep, no size effects), the model and thus the porous structures capture most part of the observed effects. It should be noted that the porosity for S7 in the simulated model is $\approx 27 \%$ which is smaller than the porosity obtained from the experimental data $(\approx 32 \%)$.

\subsection{Evaluation of elastic and plastic strain}

The average values for elastic (EE33) and plastic strain (PE33) along the pulling directions (averaged over the whole integration points in the silver phase) are extracted from the simulation outputs for samples $S 1(p=47 \%)$ and $S 8(p=22 \%)$ and the variation as a function of the applied strain is presented in Fig. $8 a$ and b. In sample S1, by applying a macroscopic strain of about $0.9 \%$ (equivalent to a stress of $35 \mathrm{MPa}$ ) the generated average elastic (blue 

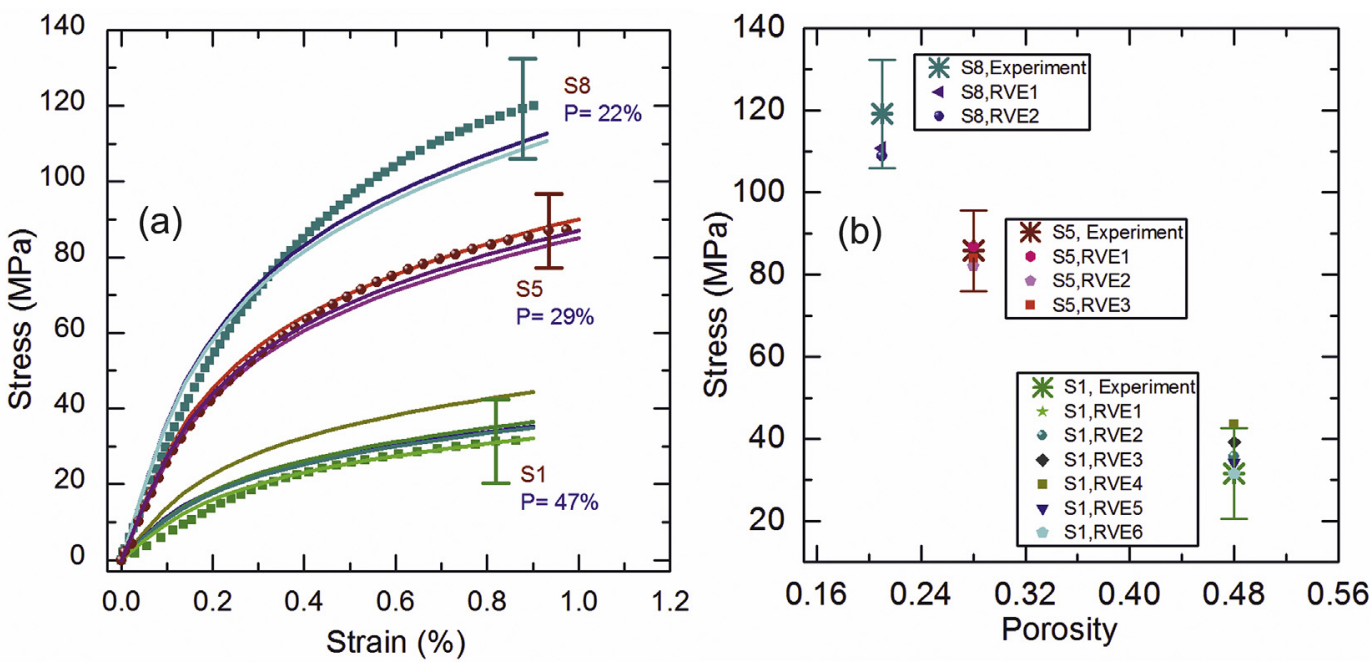

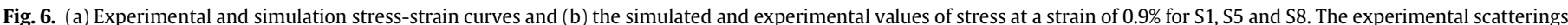
are presented as error bars.
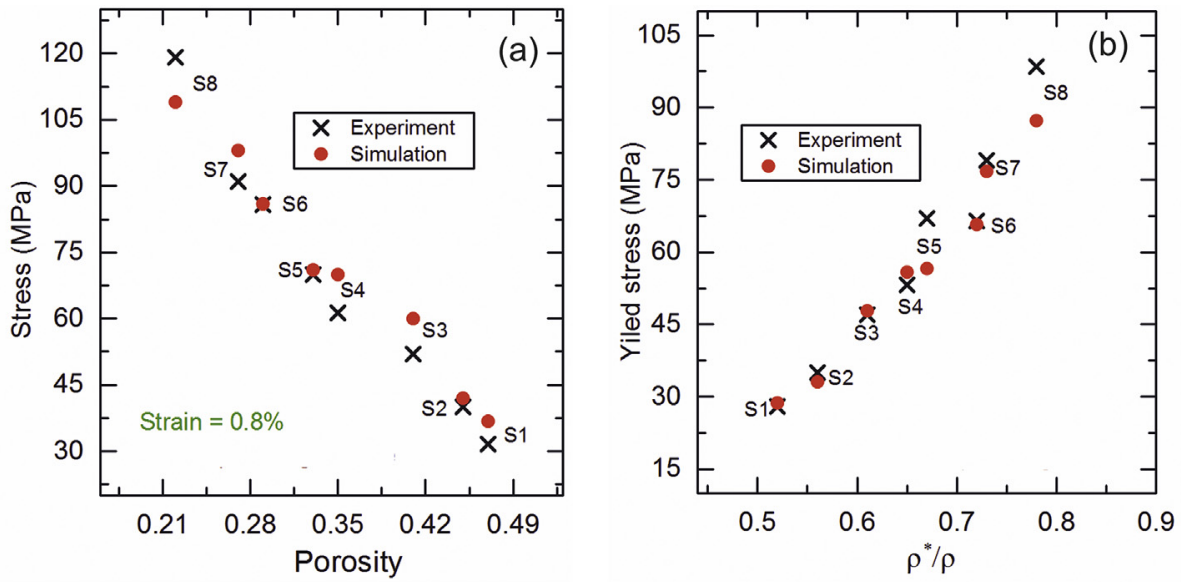

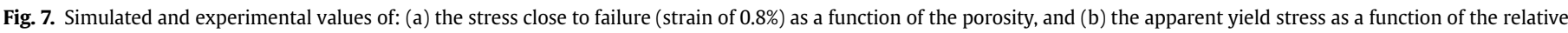
density $\left(\rho^{*} / \rho\right)$ for eight samples.
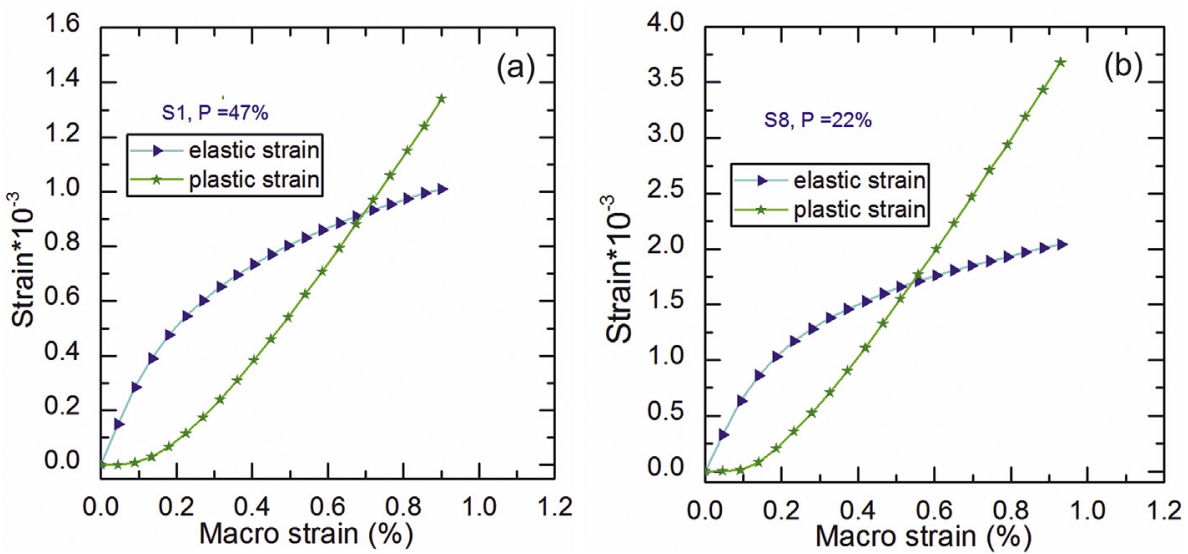

Fig. 8. Variation of the micro scale elastic and plastic strain along the pulling direction (obtained from simulation) as a function of the applied strain for: (a) S1, (b) S8.

triangle) and plastic strains (green stars) in the pulling direction are $0.10 \%$ and $0.13 \%$ respectively, with a total of $0.23 \%$. In sample S8, for the same macroscopic strain corresponding to a stress of $110 \mathrm{MPa}$, the elastic and plastic strains are $0.2 \%$ and $0.37 \%$ respectively. The results show that, for a given applied strain, the generated elastic and plastic strains strongly depend on the material porosity and 
that the elastic-to-plastic strain ratio increases with porosity: the ratio of the elastic-to-plastic strain (EE33/PE33) in S1 and S8 are 0.75 and 0.54 respectively. The structure is more compliant in the sample with higher porosity and as a consequence deforms more elastically upon applying stress and therefore less plastic strain need to be generated to reach a similar level of imposed strain.

The heterogeneity (or scattering) of the elastic strain inside samples S1 and S8 is evaluated as the standard deviation of the elastic strain EE3 at the integration points, its evolution as a function of the applied stress is presented in Fig. 9. A quasi linear behavior is observed for both specimens; note that the slope of the high porous material is significantly sharper. By increasing the applied stress from 0 to $42 \mathrm{MPa}$ the elastic strain scattering increase from 0 to $0.09 \%$ in S1 (green spheres), wherein in S8 by applying a stress around $110 \mathrm{MPa}$ the elastic strain scattering reaches $0.086 \%$ (red diamonds). Elastic strain heterogeneity is directly correlated to the stress heterogeneity and, for a linear elastic system, linearly proportional to the average strain, average stress and thus also to the applied stress. In Fig. 9 a quasi-linear trend is observed for the porous samples suggesting (for the presented strain range) that plasticity does not induce significant local stress redistribution in this model; a strong stress redistribution would affect the stress heterogeneity causing a nonlinear variation of the elastic strain scattering with the applied stress.

Fig. 10a and b displays the simulated elastic strain scattering (purple rectangles) and the experimental RMS strains for samples S1 and S8 obtained from the (111) (green stars) and (200) (blue spheres) plane families as function of the applied stress. The RMS strain is extracted from the change in diffraction peak broadening during tensile deformation using the $\mathrm{W}-\mathrm{H}$ deconvolution method.

A linear behavior is observed for the simulated elastic strain scattering. However for both experimental specimens, two distinct regions can be identified. Initially the RMS stays more or less constant, while after an applied stress of $5 \mathrm{MPa}$ in S1 and $25 \mathrm{MPa}$ in S8 a non-linear increase is observed. This initial offset could be related to residual stresses and/or primary creep which are not accounted for in the simulation. In sample S1, the experimental RMS strain for the (200) approaches the simulated elastic strain scattering. In sample S8 the experimental RMS strain for the (200) becomes larger than the simulation values once the applied stress increases to $60 \mathrm{MPa}$. The present model tends to underestimate the strain heterogeneity as neither the grain structure nor the crystal plastic anisotropy are considered in the simulations.

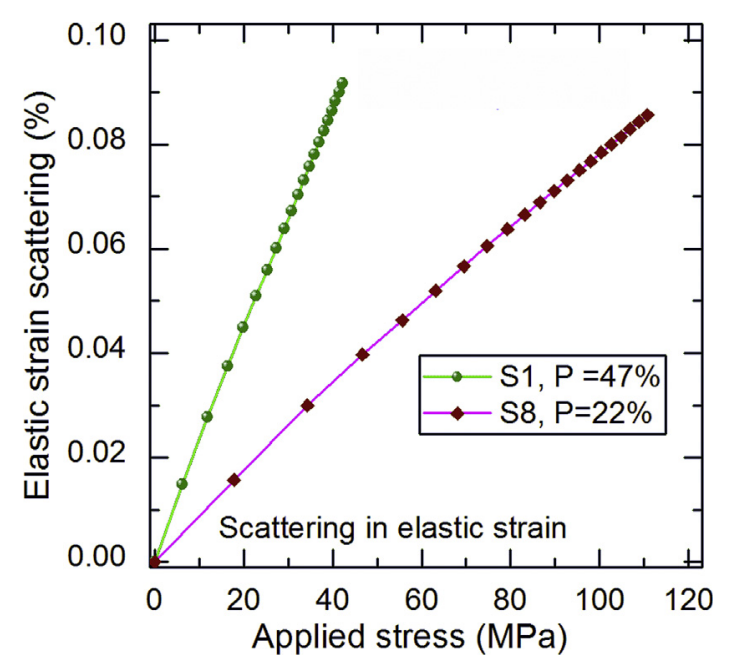

Fig. 9. Elastic strain scattering of the integration points of S1 and S8 as a function of applied stress.
The equivalent plastic strain (PEEQ) averaged over all integration points inside the silver skeleton is calculated from the simulation outputs for S1, S5 and S8 and its variation is presented in Fig. 11 as function of (a) applied strain and (b) applied stress. PEEQ is the scalar measure of the strain corresponding with the Mises stress which is generated inside the ligaments. According to Fig. 11, for an imposed strain of $0.8 \%$ a larger plastic strain is accumulated inside the samples with lower porosity; the generated PEEQ strain equals $0.16 \%$ for S1, $0.35 \%$ for S5 and $0.39 \%$ for S8. This trend is directly related to the higher elastic compliance of specimens with higher porosity. On the other hand, the variation of the plastic strain with stress [Fig. 11b] indicates that the plastic strain accumulates faster with higher porosity. This behavior can be explained by the strong stress amplification caused by higher porosity. It should be noted here that despite being stronger in terms of engineering yield stress and ultimate stress, lower porosity silver layers would thus accumulate more plastic strains under displacement controlled conditions, which could generate earlier damage and failures in fatigue-cyclic loading.

2D PEEQ distributions for S1 and S8 in the macroscopically elastic regime (at a macro strain around $0.0045 \%$ ) are shown in Fig. 12. The distribution corresponds to a cutting plane view in $X-Y$ with applied displacement along Z-axis. The pores are shown with black color. Plastic strain zones reaching values well above $0.05 \%$ are observed in the vicinity of the pores and inside the finer and more curved ligaments. The creation of the plastic zone while the whole specimen is macroscopically in the elastic regime emphasizes the importance of the heterogeneity of the porous structures, leading to highly non-uniform stress distributions and consequently early local plastic deformation and stress amplifications in the microstructure of the samples.

\subsection{Local stress inside the silver ligaments}

The average effective stress acting on the reduced cross section of the porous material is estimated by dividing the applied stress $\sigma^{*}$ (at the macroscale) by the relative density $\rho^{*} / \rho$. The reliability of this estimation on the average local (microscale) stress is verified with the help of the simulations. For this purpose, the volume averaged value of the stress along the pulling direction $\left(\sigma_{33}\right)$ extracted from simulation is compared with the average effective stress estimated as $\sigma^{*} /\left(\rho^{*} / \rho\right)$. Fig. 13 displays the comparison between these two stress measures for sample (a) S1 and (b) S8. A good agreement is observed for $S 8$, however in sample $S 1 \sigma^{*} /\left(\rho^{*} / \rho\right)$ deviates up to $6 \%$ from $\sigma_{33}$.

Nevertheless we conclude that dividing the applied stress by relative density $\sigma^{*} /\left(\rho^{*} / \rho\right)$ is an appropriate method for estimation of the average longitudinal stress inside the silver ligaments.

In Fig. 14a the values of the average local stress $\left[\sigma^{*} /\left(\rho^{*} / \rho\right)\right]$ obtained from the simulation at macroscopic yield point $(0.2 \%$ plastic strain) is plotted versus the porosity. A decrease in the local stress at yield is observed by increasing the porosity of the specimens. Fig. 14b shows the variation of the averaged PEEQ inside the ligaments of the specimens as a function of the porosity at a macrostrain of $0.85 \%$ (close to failure). Increasing the porosity of the samples leads to a decrease in the accumulated plastic strain inside the ligaments.

\section{Discussion}

For the sample with highest porosity (S1) a scattering of $11 \%$ in flow stress is obtained depending on the choice of the RVE in spite that these RVEs have similar averaged porosity. This suggests that other parameters such as pore morphology, pore size distribution, shape and size distribution of the silver ligaments also play a role in 

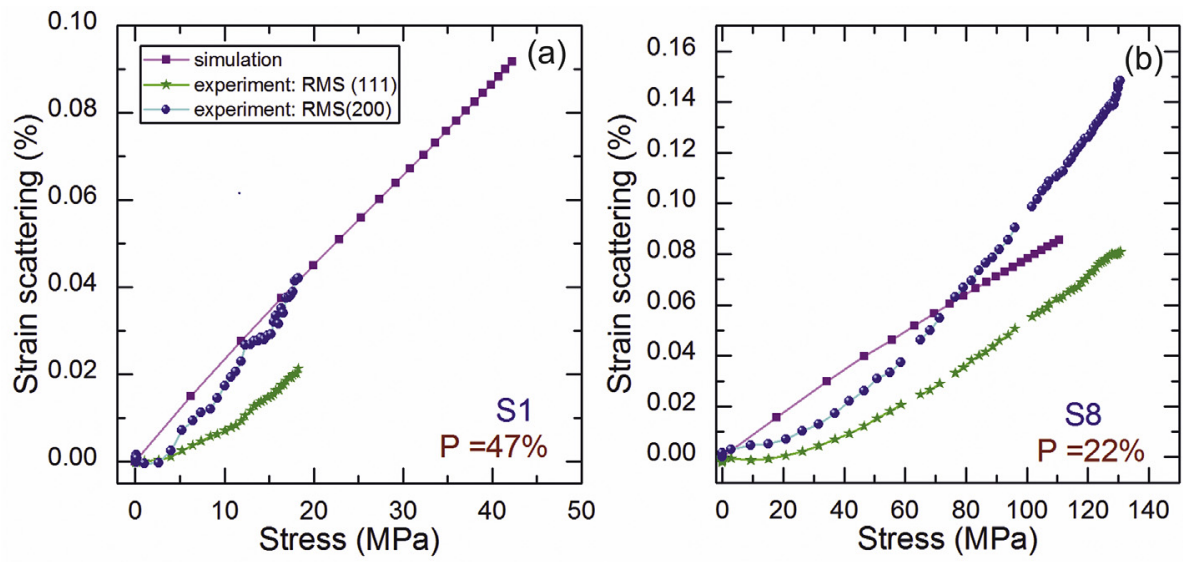

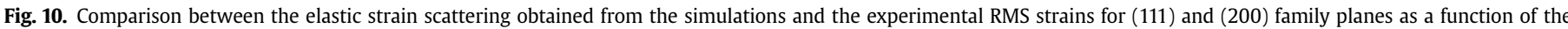
applied stress for: (a) S1 and (b) S8 deformed during continuous tensile test at a strain rate of $0.01 \% . \mathrm{s}^{-1}$.

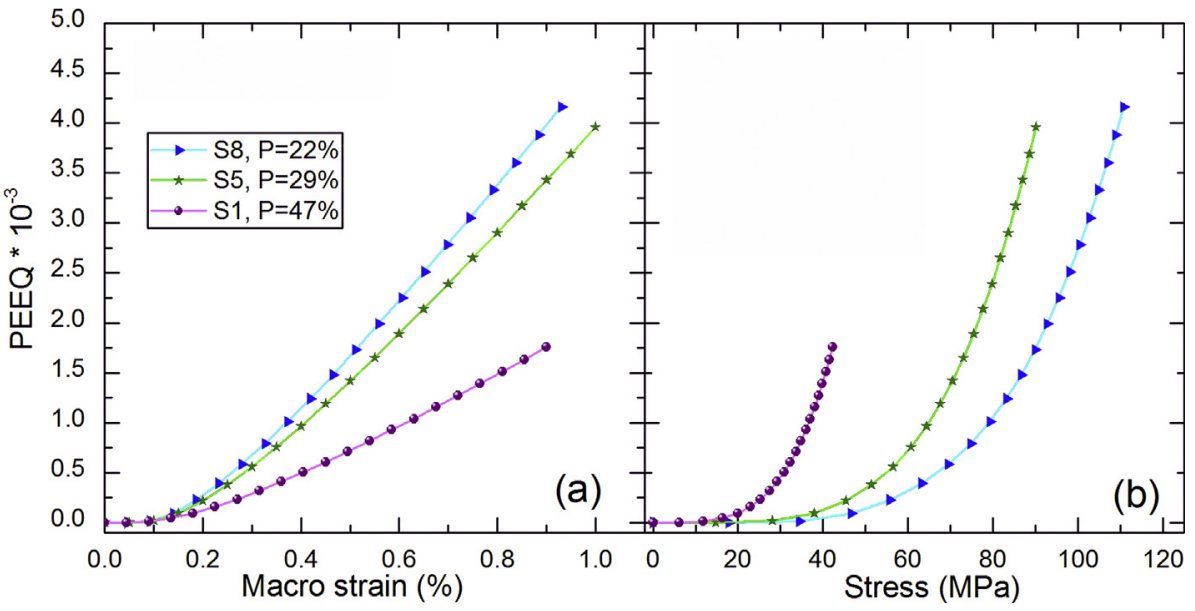

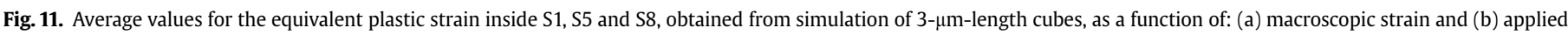
stress.
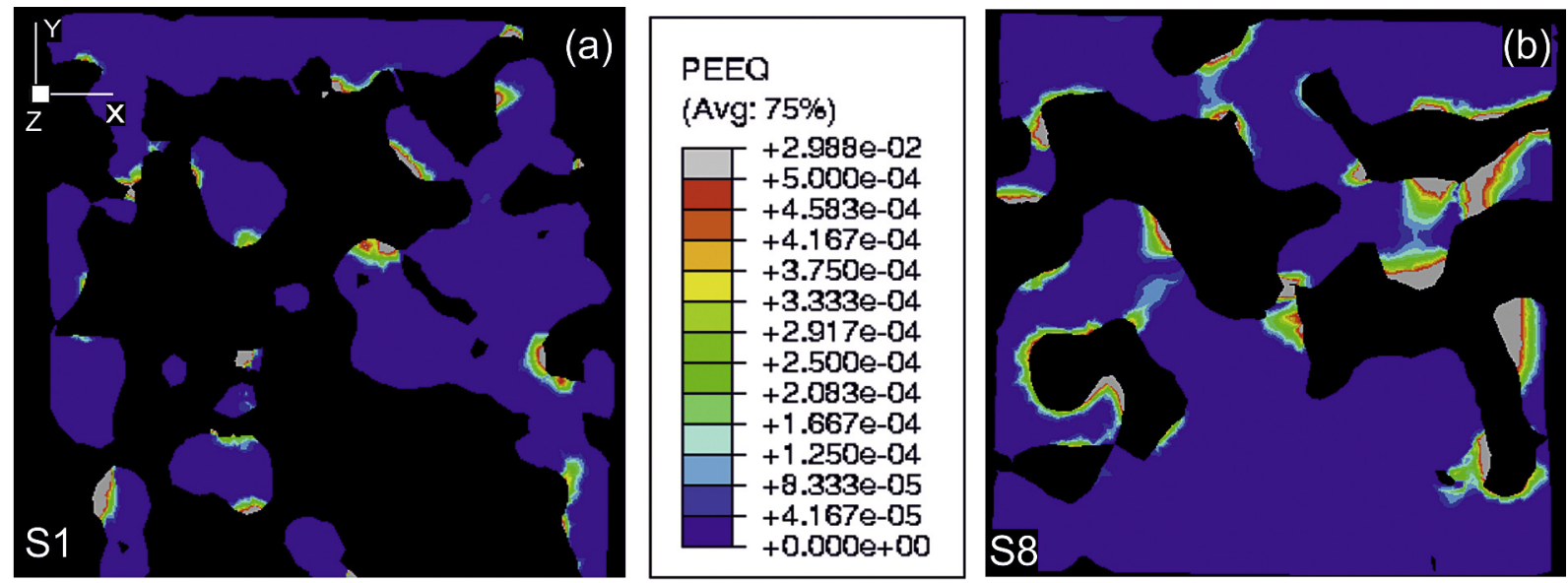

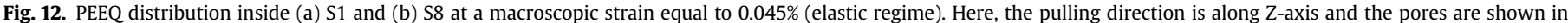
black color. Plastic strain above $0.05 \%$ is represented in grey.

the mechanical properties and might have evolved on length scales larger than the estimated RVE size of $3 \mu \mathrm{m}$. Comparison between the simulations (averaged over several RVEs) and experiments reveals that the model captures the general plastic behavior and can predict the flow stress of the samples with a deviation less than $20 \%$ (Fig. 7). This difference is surprisingly small, given the large 

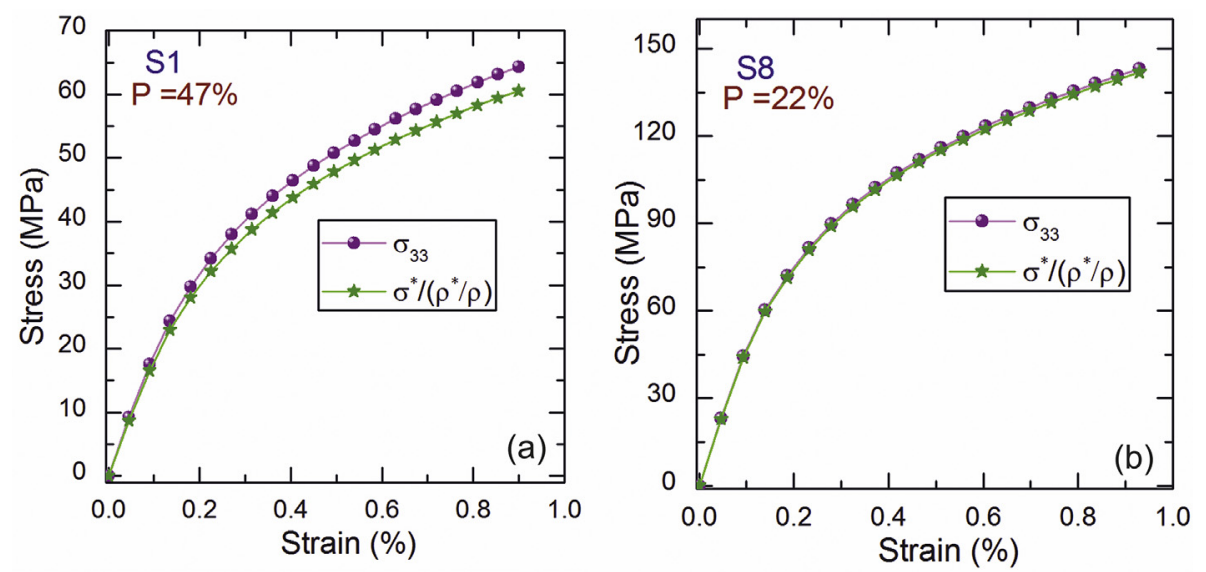

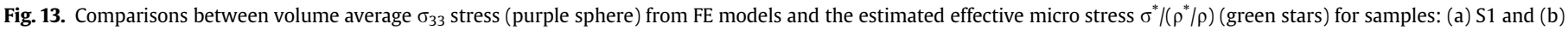

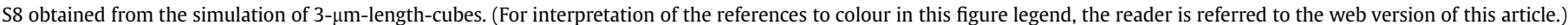
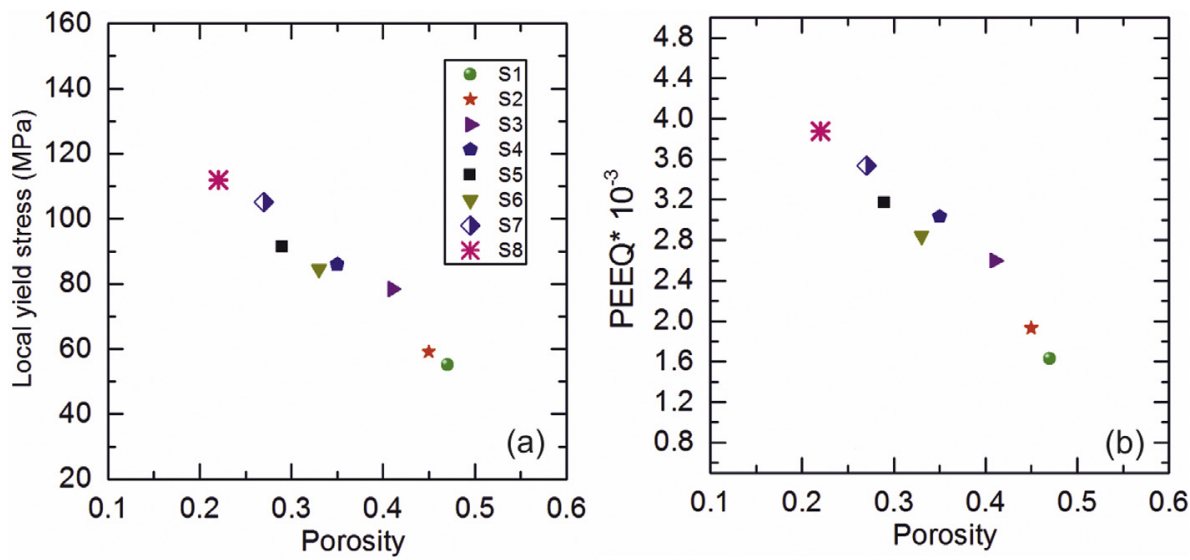

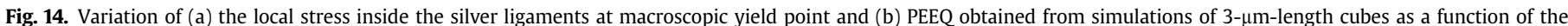
porosity at an applied macrostrain of $0.85 \%$.

simplifications of the model: the model is isotropic, homogeneous, free of residual stresses, rate independent, and various microstructural features (grains, grain boundaries, defects) have been omitted. This implies that a large part of the mechanical behavior is controlled by the porosity, porous morphology and size distribution of the silver ligaments, while the pre-existing defects [14], grain structure, grain size and grain boundaries have only a second order of the importance.

The analysis of the average elastic (EE33) and plastic strain (PE33) shows that, for the same applied macroscopic tensile strain, the ratio of the elastic to plastic strain is higher with increasing porosity (Fig. 8). This behavior is in good agreement with variation of the local stress at macroscopic yield and the average plastic strain inside silver ligaments with porosity. According to Fig. 14, increasing the porosity of the samples leads to a decrease in average local stress at yield stress as well as in the accumulated plastic strain inside the ligaments. This behavior is attributed to the porous structure: the beam-like structure formed by silver ligaments inside the highly porous samples is more compliant and can develop more bending deformation during tensile loading, which is coherent with the observed reduction of apparent elastic modulus. As a consequence, for a given applied macroscopic strain, elastic deformation of the highly porous specimens is larger and thus less plasticity is required to accommodate the prescribed strain. On the other hand, the silver ligaments inside the low porous materials are more constraint. Consequently, a larger stress is required to align them along the applied stress, which results in a larger induced plastic strain inside the ligaments.

The results from ptychographic-tomography indicate a nonuniform pore distribution inside the sintered silver layers [15]. The large heterogeneity in the porous structures results in a nonuniform local stress distribution. This is explained by the stress amplification factor, which relates the micro (local) stress to the applied stress. As reported in the literature [30-32], the pores increase not only the average but also the peak stress amplification factor leading to larger local stresses in their vicinity; the stress amplification factor depends on the size and the shape of the pores [30-32]. A non-uniform stress distribution inside the sample results in a non-uniform elastic and plastic strain distribution. Increasing the porosity of the sample (such as in S1), increases the stress distribution heterogeneity leading to a larger scattering in the elastic strain inside the ligaments compared to the low porosity sample (S8). This explains the steeper slope of the elastic strain scatter versus applied stress of sample S1 compared to S8 as presented in Fig. 9. Furthermore, the high stress concentrations caused by the ligament geometry (outer surface of ligaments in curved regions) can also generate local plastic deformation (plastic zones) inside the ligaments as it is visible in Fig. 12.

Comparing the elastic strain scattering obtained from the simulations with the experimental RMS strain of (111) and (200) family 
planes (Fig. 10) reveals important differences between the samples. The experimental RMS strain seems to grow slowly in the early stage of the loading for both specimens and differs significantly from the simulation. The lower values of RMS obtained from the experiments may be a sign for creep or residual stresses redistribution which are not considered in the simulation model. Since the model is rate independent it would overestimate stress and strain inhomogeneity at low strain rates. On the other hand by increasing the applied stress (above $5 \mathrm{MPa}$ for S1 and $25 \mathrm{MPa}$ for S8) a faster increase in experimental RMS strain is observed. In sample S1 the experimental RMS [Fig. 10a] approaches the simulated values. This suggests that in highly porous samples a large portion of the experimental RMS strain (and thus peak broadening) is originating from the heterogeneity in the porous structure which causes a nonuniform elastic strain distribution inside the sample and the local elastic bending of the ligaments. On the other hand, in S8, [Fig. 10b], the RMS in (200) planes exceeds the elastic strain scattering obtained from the simulation after an applied stress of $60 \mathrm{MPa}$. The larger value of the experimental RMS can be explained by microstructural heterogeneity due to grain structures and intra-granular dislocations, parameters not taken into account in the current simulation model.

\section{Conclusions}

Microstructure-based finite element simulations are performed in order to make a correlation between the mechanical behavior and the porous structures. The simulations are applied on 3- $\mu \mathrm{m}-$ side-length cubes extracted from 3D ptychographic tomography images meshed by quadratic ten-node tetrahedrons. The model considers an isotropic elastoplastic behavior with von-Mises plasticity without creep for the silver phase. The influence of the grain size, grain boundary character and pre-existing defects is neglected. The simulation model can capture the general plasticity and predict the mechanical behavior with a deviation less than $20 \%$. This good agreement between the simulation and the experiment indicate that the pores are the main factor in controlling the flow stress and plasticity of the specimens.

In the sample sintered at the lowest pressure/temperature/time which has the highest porosity, the elastic-to-plastic strain ratio is highest and less plastic strain is accumulated inside the ligaments for a given applied strain. The scattering in elastic strain inside the silver ligaments is also higher. The analysis of the local stress and plastic strain versus porosity suggests that, the plastic deformation of silver ligaments is mainly caused by local stress concentrations at highly curved ligaments and local bending deformation during their alignment along the applied load. On the other hand, in low porosity samples, the porous skeleton is stiffer and the microstructure is more uniform. Therefore, the development of the plastic strain inside the silver ligaments is more pronounced.

Finally, a comparison between the elastic strain scattering and experimental RMS strains suggests that in highly porous samples (S1), a large part of the diffraction peak broadening during tensile loading originates from the porous structure heterogeneity and local elastic bending of the ligaments. In low porosity samples (S8) grain morphologies and intra-granular defects such as dislocations can form additional sources of the peak broadening during the deformation which are not captured in the present modeling approach.

\section{Acknowledgements}

This work has been financially supported by the Swiss Nanoscience Institute (SNI) in Basel. The authors would like to thank Dr. A. Diaz and Dr. M. Holler for their help in experimental parts of the project.

\section{References}

[1] H. Altenbach, A. Öchsner (Eds.), Cellular and Porous Materials in Structures and Processes, Springer Vienna, Vienna, 2010 (Accessed 6 February 2016), http://link.springer.com/10.1007/978-3-7091-0297-8.

[2] Sir William Thomson, On the division of space with minimum partitional area, Philos. Mag. 24 (151) (1887) 503 (n.d.).

[3] R. Gabbrielli, A.J. Meagher, D. Weaire, K.A. Brakke, S. Hutzler, An experimental realization of the Weaire-Phelan structure in monodisperse liquid foam Philos. Mag. Lett. 92 (2012) 1-6, http://dx.doi.org/10.1080/ 09500839.2011.645898.

[4] K.A. Brakke, The surface evolver, Exp. Math. 1 (1992) 141-165, http:// dx.doi.org/10.1080/10586458.1992.10504253.

[5] A.M. Kraynik, D.A. Reinelt, F. van Swol, Structure of random monodisperse foam, Phys. Rev. E 67 (2003) 31403, http://dx.doi.org/10.1103/ PhysRevE.67.031403.

[6] Z. Chen, X. Wang, F. Giuliani, A. Atkinson, Analyses of microstructural and elastic properties of porous SOFC cathodes based on focused ion beam tomography, J. Power Sources 273 (2015) 486-494, http://dx.doi.org/10.1016/ j.jpowsour.2014.09.131.

[7] K. Gao, V. Dommelen, J.A. W, M.G.D. Geers, P. Göransson, Microstructurebased numerical modelling of foams for acoustic shielding, 2014, pp. 881-887 (Accessed 16 February 2016), http://www.diva-portal.org/smash/record.jsf? pid=diva2\%3A806518\&dswid $=5765$.

[8] M.A. Knackstedt, C.H. Arns, M. Saadatfar, T.J. Senden, A. Limaye, A. Sakellariou, A.P. Sheppard, R.M. Sok, W. Schrof, H. Steininger, Elastic and transport properties of cellular solids derived from three-dimensional tomographic images, Proc. R. Soc. Lond. Math. Phys. Eng. Sci. 462 (2006) 2833-2862, http:// dx.doi.org/10.1098/rspa.2006.1657.

[9] J. Carr, X. Milhet, P. Gadaud, S.A.E. Boyer, G.E. Thompson, P. Lee, Quantitative characterization of porosity and determination of elastic modulus for sintered micro-silver joints, J. Mater. Process. Technol. 225 (2015) 19-23, http:// dx.doi.org/10.1016/j.jmatprotec.2015.03.037.

[10] R.A. Hardin, C. Beckermann, Effect of porosity on the stiffness of cast steel Metall. Mater. Trans. A 38 (2007) 2992-3006, http://dx.doi.org/10.1007/ s11661-007-9390-4.

[11] W. Jiang, Z. Cao, X. Sun, H. Liu, Three-dimensional microstructure reconstruction and finite element simulation of gas pores in the high-pressure diecasting AZ91 Mg alloy, Microsc. Microanal. Off. J. Microsc. Soc. Am. Microbeam Anal. Soc. Microsc. Soc. Can. 21 (2015) 1420-1425, http://dx.doi.org/10.1017/ S1431927615015172.

[12] N. Michailidis, F. Stergioudi, H. Omar, D.N. Tsipas, An image-based reconstruction of the 3D geometry of an Al open-cell foam and FEM modeling of the material response, Mech. Mater 42 (2010) 142-147, http://dx.doi.org/ 10.1016/j.mechmat.2009.10.006.

[13] W. Denk, H. Horstmann, Serial block-face scanning electron microscopy to reconstruct three-dimensional tissue nanostructure, PLoS Biol. 2 (2004) e329, http://dx.doi.org/10.1371/journal.pbio.0020329.

[14] S. Zabihzadeh, S. Van Petegem, L.I. Duarte, R. Mokso, A. Cervellino, H. Van Swygenhoven, Deformation behavior of sintered nanocrystalline silver layers, Acta Mater 97 (2015) 116-123, http://dx.doi.org/10.1016/ j.actamat.2015.06.040.

[15] S. Zabihzadeh, S. Van Petegem, M. Holler, A. Diaz, L.I. Duarte, H. Van Swygenhoven, Deformation behavior of nanoporous polycrystalline silver. Part I: microstructure and mechanical properties, Acta Mater. 131 (2017) 467-474, http://dx.doi.org/10.1016/j.actamat.2017.04.021.

[16] P.R. Willmott, D. Meister, S.J. Leake, M. Lange, A. Bergamaschi, M. Böge, M. Calvi, C. Cancellieri, N. Casati, A. Cervellino, Q. Chen, C. David, U. Flechsig F. Gozzo, B. Henrich, S. Jäggi-Spielmann, B. Jakob, I. Kalichava, P. Karvinen, J. Krempasky, A. Lüdeke, R. Lüscher, S. Maag, C. Quitmann, M.L. ReinleSchmitt, T. Schmidt, B. Schmitt, A. Streun, I. Vartiainen, M. Vitins, X. Wang, $\mathrm{R}$. Wullschleger, The materials science beamline upgrade at the Swiss light source, J. Synchrotron Radiat. 20 (2013) 667-682, http://dx.doi.org/10.1107/ S0909049513018475.

[17] F. Gozzo, B. Schmitt, T. Bortolamedi, C. Giannini, A. Guagliardi, M. Lange, D. Meister, D. Maden, P. Willmott, B.D. Patterson, First experiments at the Swiss light source materials science beamline powder diffractometer, J. Alloys Compd. 362 (2004) 206-217, http://dx.doi.org/10.1016/S0925-8388(03) 00585-1.

[18] C.E. Kril, R. Birringer, Estimating grain-size distributions in nanocrystalline materials from X-ray diffraction profile analysis, Philos. Mag. A 77 (1998) 621-640, http://dx.doi.org/10.1080/01418619808224072.

[19] S. Brandstetter, Deformation mechanisms of nanocrystalline nickel studied by in-situ X-ray diffraction, École Polytechique Fédérale De Lausanne (2008) (n.d.).

[20] S. Brandstetter, P.M. Derlet, S. Van Petegem, H. Van Swygenhoven, Williamson-Hall anisotropy in nanocrystalline metals: X-ray diffraction experiments and atomistic simulations, Acta Mater 56 (2008) 165-176, http:// dx.doi.org/10.1016/j.actamat.2007.09.007.

[21] P.A. Yushkevich, J. Piven, H.C. Hazlett, R.G. Smith, S. Ho, J.C. Gee, G. Gerig, Userguided 3D active contour segmentation of anatomical structures: significantly improved efficiency and reliability, NeuroImage 31 (2006) 1116-1128, http:// 
dx.doi.org/10.1016/j.neuroimage.2006.01.015.

[22] J. Cugnoni, M. Galli, Representative volume element size of elastoplastic and elastoviscoplastic particle-reinforced composites with random microstructure, CMES Comput. Model. Eng. Sci. 66 (2010) 165-186, http://dx.doi.org/ 10.3970/cmes.2010.066.165.

[23] M. Maleki, J. Cugnoni, J. Botsis, Multi-scale modeling of elasto-plastic response of $\mathrm{SnAgCu}$ lead-free solder alloys at different ageing conditions: effect of microstructure evolution, particle size effects and interfacial failure, Mater. Sci. Eng. A 661 (2016) 132-144, http://dx.doi.org/10.1016/ j.msea.2016.03.011.

[24] H. Shen, L.C. Brinson, Finite element modeling of porous titanium, Int. J. Solids Struct. 44 (2007) 320-335, http://dx.doi.org/10.1016/j.ijsolstr.2006.04.020.

[25] W.J. Drugan, J.R. Willis, A micromechanics-based nonlocal constitutive equation and estimates of representative volume element size for elastic composites, J. Mech. Phys. Solids 44 (1996) 497-524, http://dx.doi.org/10.1016/ 0022-5096(96)00007-5.

[26] Simulation Numérique de Traction de Matériaux Poreux, De Gol Philippe, 2012 (n.d.)
[27] M. Galli, J. Cugnoni, J. Botsis, J. Janczak-Rusch, Identification of the matrix elastoplastic properties in reinforced active brazing alloys, Compos. Part Appl. Sci. Manuf. 39 (2008) 972-978, http://dx.doi.org/10.1016/ j.compositesa.2008.03.007.

[28] F.R.F.D.R. Smith, Low-temperature properties of silver, J. Res. Natl. Inst. Stand. Technol. 100 (1995), http://dx.doi.org/10.6028/jres.100.012.

[29] W.F. Hosford, Strain Hardening of Metals, in: Solid Mech, Cambridge University Press, 2010, http://dx.doi.org/10.1017/CBO9780511841422.005.

[30] X. Markenscoff, Stress amplification in the neighborhood of an eccentric large hole in a strip in tension, Z. Für Angew. Math. Phys. ZAMP 51 (2000) 550-554, http://dx.doi.org/10.1007/s000330050016.

[31] V.A. Lubarda, X. Markenscoff, Stress magnification due to stretching and bending of thin ligaments between voids, Arch. Appl. Mech. 76 (2006) 295-310, http://dx.doi.org/10.1007/s00419-006-0028-6.

[32] I. Tsukrov, M. Kachanov, Stress concentrations and microfracturing patterns for interacting elliptical holes, Int. J. Fract. 68 (1994) R89-R92, http:/ dx.doi.org/10.1007/BF00013078. 